\title{
COLD MATERIAL IN DISTANT RADIO GALAXIES
}

\author{
HUUB RÖTTGERING \\ MRAO/IoA, Cambridge, UK
}

\begin{abstract}
Dust, H I and molecular gas must be important components of distant radio galaxies. Recent observations to detect these constituents are discussed.
\end{abstract}

\section{Introduction}

Radio galaxies are important probes of the early universe. During the last few years extensive surveys for distant radio galaxies have been carried out using different selection techniques (eg. McCarthy 1993; Miley 1994). At the moment more than 60 radio galaxies are known at redshifts larger than two. The main advantage of studying these galaxies, as opposed to studying other objects known at $z>2$, such as absorption systems and quasars, is that radio galaxies contain three extended components that are easily resolved with conventional techniques. These components are the radio synchrotron plasma, the ionised gas and the IR/optical/UV continua. Studying these components and their relation to one another has revealed a great deal about the nature of these systems. One of the most interesting parameters is the maturity of the stellar population. Unfortunately, it is difficult to use the observations of the three different components just mentioned to probe the stellar content directly. The main reason for this is that it is difficult to determine the relative contribution from stars and non-thermal emission from the IR/optical/UV continuum data. In nearby galaxies an important probe of star formation is molecular gas. The sensitivity of present day instruments is good enough that at least attempting to detect cold material in distant radio galaxies seems a worthwhile exercise. 


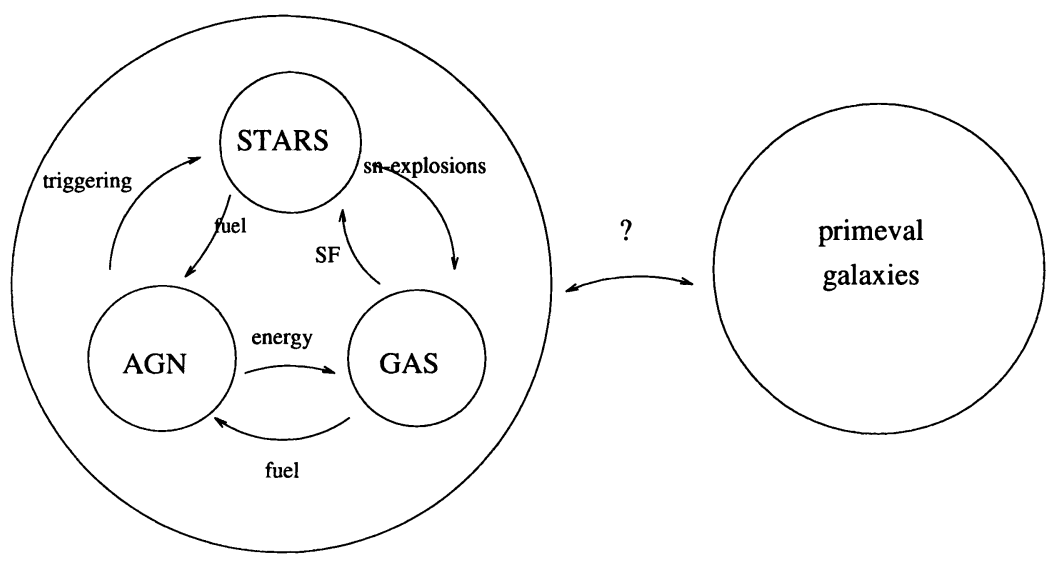

Figure 1. This diagram shows schematically the relation between the various constituents together with their interactions within a distant radio galaxy. One of the main questions is what such systems tell us about primeval galaxies.

\section{The importance of cold gas in distant radio galaxies}

Figure 1 is an illustration of how complicated the systems we call distant radio galaxies are. The three main constituents of these objects are the active nucleus, stars and gas in various phases. All three constituents are related to one another. It is clear that the stars in these systems have to be formed from gas and that stellar mass loss, mostly in the form of supernovae explosions, will transfer material from stars back to the ISM. This symbiotic relation between stars and gas is complicated by the presence of the AGN. The AGN is being fuelled by surrounding material (eg. gas/stars) and as a result it inputs energy in various forms to the gas. In some models of distant radio sources it is assumed that the radio jet emerging from the AGN triggers star formation in the ISM.

It is clear that we have to understand these processes in a certain amount of detail before we can decide what the relation is between distant radio galaxies and young and forming galaxies. Questions that should be answered include how profound the impact of the AGN is on these objects and what age these stellar systems are.

Cold material such as dust and cold gas $\left(T \ll 10^{4} \mathrm{~K}\right)$ must play an important role in these systems. Since in some models the radio galaxies are producing stars at a prodigious rate, this is the phase of the ISM that provides the building material for stars. In nearby AGN such as Seyferts the observation of molecular material shows that a large fraction of the CO gas, for example, is concentrated within one kpc of the active nucleus. This suggests that at least some of the molecular gas will ultimately fuel the AGN. 
In the rest of this contribution I will concentrate on the observational evidence for this gas phase in distant radio galaxies.

\section{Dust}

There are several ways of probing the dust content in high redshift radio galaxies. One of the first attempts was to measure the $\mathrm{Ly} \alpha / \mathrm{H} \alpha$ ratio. For two radio galaxies McCarthy et al. (1992) found values within a factor of 2 of case $B$ recombination, implying $E(B-V)=0.1$. Cimatti et al. (1993) found dust masses of order $10^{8} M_{\odot}$ from modelling the optical polarisation of radio galaxies. The most spectacular measurement of dust is probably the detection of submillimetre emission from the radio galaxy $4 \mathrm{C} 41.17(\mathrm{z}=3.8)$ implying dust masses of order a few times $10^{8} M_{\odot}$ (Dunlop et al. 1994). Van Ojik et al. (1994) discovered that the radio galaxy 0211-122 $(z=2.34)$ has a peculiar emission line spectrum, with Ly $\alpha$ very much fainter with respect to the high ionisation lines than in typical high redshift radio galaxies. They suggest that this galaxy is undergoing a vigorous starburst producing enough dust to attenuate the Ly $\alpha$ emission.

\section{Neutral Hydrogen}

There are two important methods of detecting $\mathrm{H}$ I associated with radio galaxies. The first method is to measure the redshifted $21 \mathrm{~cm}$ absorption line against the radio continuum. The only distant radio galaxy for which this has been measured is $0902+34(\mathrm{z}=3.4)$. Uson et al. (1991) found an absorber in this system with a column density of $4.4 \times 10^{22}$ atoms $\mathrm{cm}^{-2}$, assuming a spin temperature of $10^{4} \mathrm{~K}$. The existence of this absorber was confirmed by Briggs et al. (1993).

The second method is to measure absorption against the Ly $\alpha$ emission of high-z radio galaxies. The main reason that this is possible is that the Ly $\alpha$ emission from distant radio galaxies can be very bright $\left(>10^{-15} \mathrm{erg} \mathrm{s}^{-1}\right)$. We therefore started a programme to detect such absorption in distant radio galaxies. The high resolution AAT spectra $(1.5 \AA)$ of the Ly $\alpha$ region of the $z=2.9$ radio galaxy $0943-242$ reveal a complex emission line profile which is dominated by a black absorption trough centered $250 \mathrm{~km} \mathrm{~s}^{-1}$ blueward of the emission peak (Figure 2, see also Röttgering et al. 1994). The main $\mathrm{HI}$ absorber has a column density of $1 \times 10^{19} \mathrm{~cm}^{-2}$.

One of the fundamental limitations in the study of quasar absorption lines is that quasars are point sources so that in general no information can be obtained about the spatial scale of the absorber. In the case of radio galaxies however, the Ly $\alpha$ emission is extended, in some cases even up to 10 arcsec. The main absorption in the Ly $\alpha$ emission from 0943-242 is black and it covers the entire Ly $\alpha$ emission region which has an angular scale of 


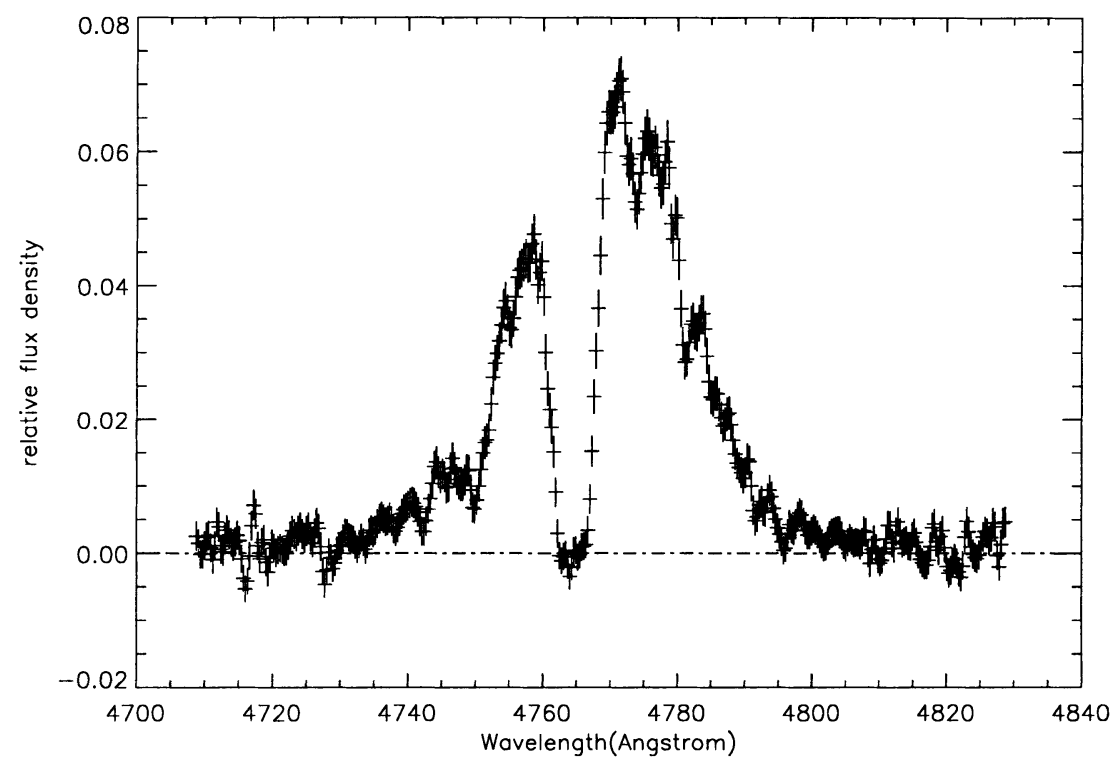

Figure 2. Part of the high resolution AAT spectrum spectra $(1.5 \AA)$ of the Ly $\alpha$ region of the $z=2.9$ radio galaxy 0943-242. (see also Röttgering et al. 1994).

1.7 arcsec. Its linear size is then at least $13 \mathrm{kpc}$, making this the first direct measurement of the spatial scale of an absorber with a column density of $\sim 10^{19} \mathrm{~cm}^{-2}$.

\section{Carbon Monoxide}

Since the CO molecule is a good tracer of star formation in galaxies, studying this molecule at cosmological distances is important for understanding the evolution of young galaxies. To date the two objects at high redshift that have a confirmed detection of a $\mathrm{CO}$ line are the ultra luminous IRAS galaxy $\mathrm{F} 10214+4724$ at $\mathrm{z}=2.3$ (Solomon et al. 1992) and the cloverleaf quasar (Barvainis et al. 1994). The good news is that these two detections demonstrate convincingly that studying $\mathrm{CO}$ in the early universe is possible. The bad news is that the IRAS galaxy F10214+4724 is the only IRAS galaxy known at these distances. Studying a sample of such objects is therefore not (yet) possible. Since the cloverleaf quasar is a gravitationally lensed quasar for which the amplification factor is not well known, the estimate for the intrinsic CO luminosity of this object is uncertain.

The significant samples of quasars and radio galaxies that exist are 
a good starting point for a further search for $\mathrm{CO}$ in the early universe. The expected width of the $\mathrm{CO}$ emission lines is a few hundred $\mathrm{km} \mathrm{s}^{-1}$ (FWHM) and the bandwidth of the receivers and backend combination as used in major sub-millimetre observatories is limited to about $1000 \mathrm{~km} \mathrm{~s}^{-1}$. It is therefore important that objects to be studied have well determined redshifts, preferably better than a few hundred $\mathrm{km} \mathrm{s}^{-1}$. Since the FWHM of the emission lines of radio galaxies is of order $1000 \mathrm{~km} \mathrm{~s}^{-1}$, a factor of $5-10$ smaller than in quasars, there is some advantage using radio galaxies rather than quasars for a survey of $\mathrm{CO}$ in the distant universe. We have started a programme to detect $\mathrm{CO}$ emission from distant radio galaxies using the JCMT and IRAM telescopes. From our spectra we have clear indications that we are indeed detecting CO lines from some of these distant objects. Currently we are working to confirm these detections using new observations. If these lines are real, they indicate that the density of CO gas is in the range $10^{4}-10^{5} \mathrm{~cm}^{-3}$ and that the temperature is of order $50-100 \mathrm{~K}$. Using galactic conversion factors the total $H_{2}$ masses are then $10^{11} M_{\odot}$, comparable to the mass obtained for F10214+4724. If correct, these detections would support the idea that distant radio galaxies are undergoing vigorous starbursts. They might, therefore, be producing the bulk of their stellar content, as expected for a forming galaxy.

Acknowledgements. I would like to thank my collaborators, Dick Hunstead, George Miley, Rob van Ojik, Paul van der Werf and Mark Wieringa for continuous discussion.

\section{References}

Barvainis, R., Tacconi, L., Antonucci, T., Alloin, D., and Coleman, P.: 1994, Nature 371, 586

Briggs, F. H., Sorar, E., and Taramopoulos, A.: 1993, ApJ 415, L99

Cimatti, A., di Serego Alighieri, S., Fosbury, R., lvati, M. S., and Taylor, D.: 1993, MNRAS 264, 264

Dunlop, J. S., Hughes, D. H., Rawlings, S., Eales, S. A., and Ward, M. J.: 1994, Nature 370,347

McCarthy, P. J.: 1993, ARA\&A 31, 639

McCarthy, P. J., Elston, R., , and Eisenhardt, P.: 1992, ApJ 387, L29

Miley, G. K.: 1994, in G. V. Bicknell, M. A. Dopita, and P. J. Quinn (eds.), The First Stromlo Conference: The Physics of Active Galaxies, Vol. 54, p. 385

Röttgering, H., Hunstead, R., Miley, G. K., van Ojik, R., and Wieringa, M. H.: 1994, Spatially Resolved Ly $\alpha$ Absorption in the $\mathrm{z}=2.9$ Radio Galaxy 0943-242, MN: submitted

Solomon, P. M., Downes, D., and Radford, S. J. E.: 1992, ApJ 398, L29

Uson, J., Bagri, D. S., and Cornwell, D. S.: 1991, Phys. Rev. Letter 67, 3328

van Ojik, R., Röttgering, H., Miley, G., Bremer, M., Macchetto, F., and Chambers, K.: 1994, A\&A 289, 54 